\title{
Composition-Dependent Oxygen Reduction Reaction Activity of Pt-Surfaced PtNi Dodecahedral Nanoframes
}

Yongchen Wang, ${ }^{1}$ Shutang Chen, ${ }^{1}$ Xudong Wang, ${ }^{1}$ Adam Rosen, ${ }^{2}$ William Beatrez, ${ }^{2}$

Lukasz Sztaberek, ${ }^{2,3}$ Haiyan Tan, ${ }^{4}$ Liang Zhang ${ }^{5, *}$, Christopher Koenigsmann, ${ }^{2, *}$ Jing Zhao $^{1, *}$

${ }^{1}$ Department of Chemistry, University of Connecticut, 55 North Eagleville Road, Storrs, CT 06269-3060 United States

${ }^{2}$ Department of Chemistry, Fordham University, 441 East Fordham Road, Bronx, NY 10458 United States

${ }^{3}$ Department of Environmental Control Technology, New York City College of Technology, 300 Jay Street, Brooklyn, NY 11201 United States

${ }^{4}$ Institute of Materials Science, University of Connecticut, Storrs, Connecticut 06269, United States

${ }^{5}$ Department of Chemical and Biomolecular Engineering, University of Connecticut, 191 Auditorium Road, Storrs, CT 06269, United States 


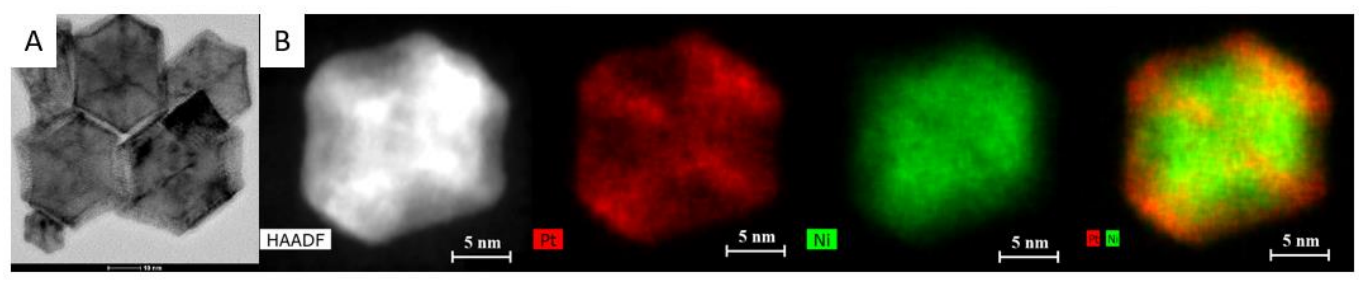

Figure S1. A) TEM images B) HAADF-STEM images and EDX mapping images of PtNi rhombic dodecahedral seed-core-frame nanostructures
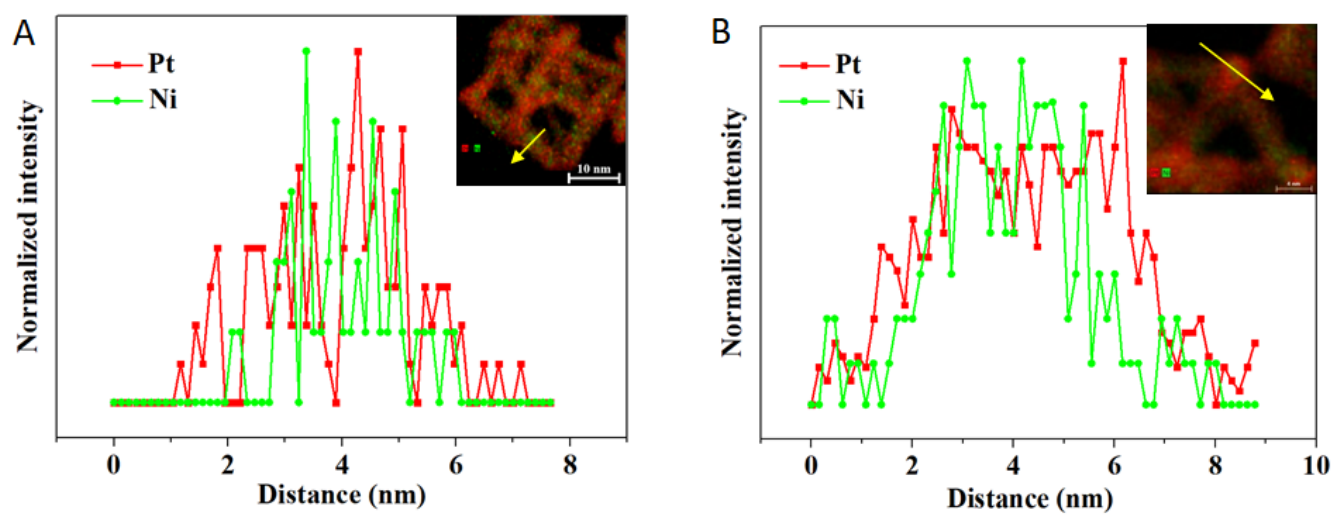

Figure S2. EDS compositional line profiles of A) an edge and B) a vertex of a nanoframe. The directions of the line profiles were plotted as arrows in the corresponding nanoframes. 


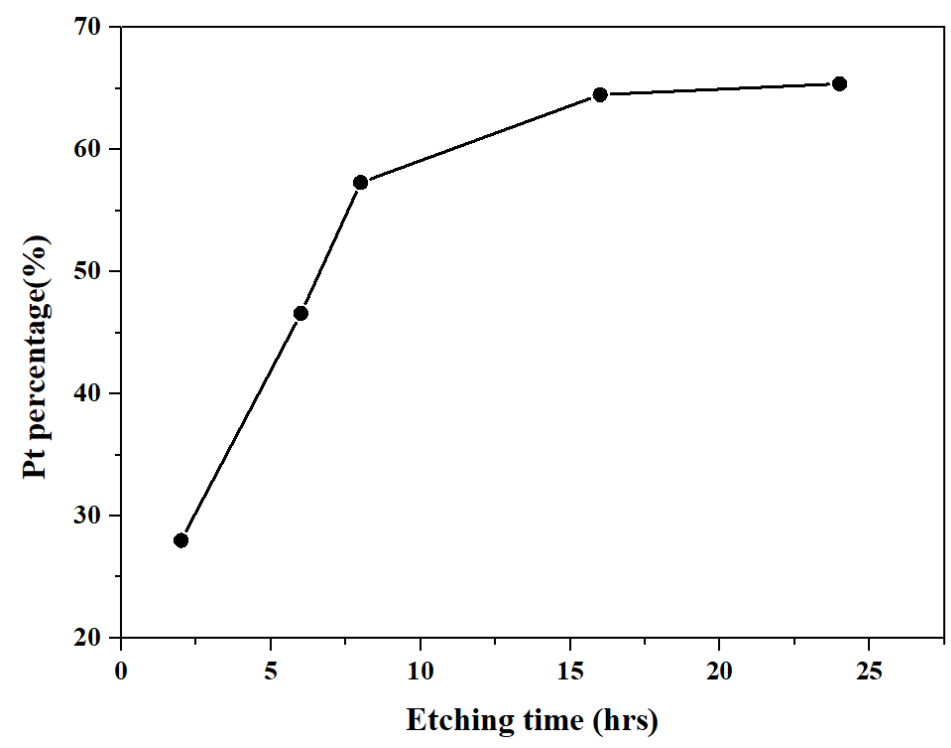

Figure S3. Composition of PtNi nanoframes determined by EDS as a function of etching time.

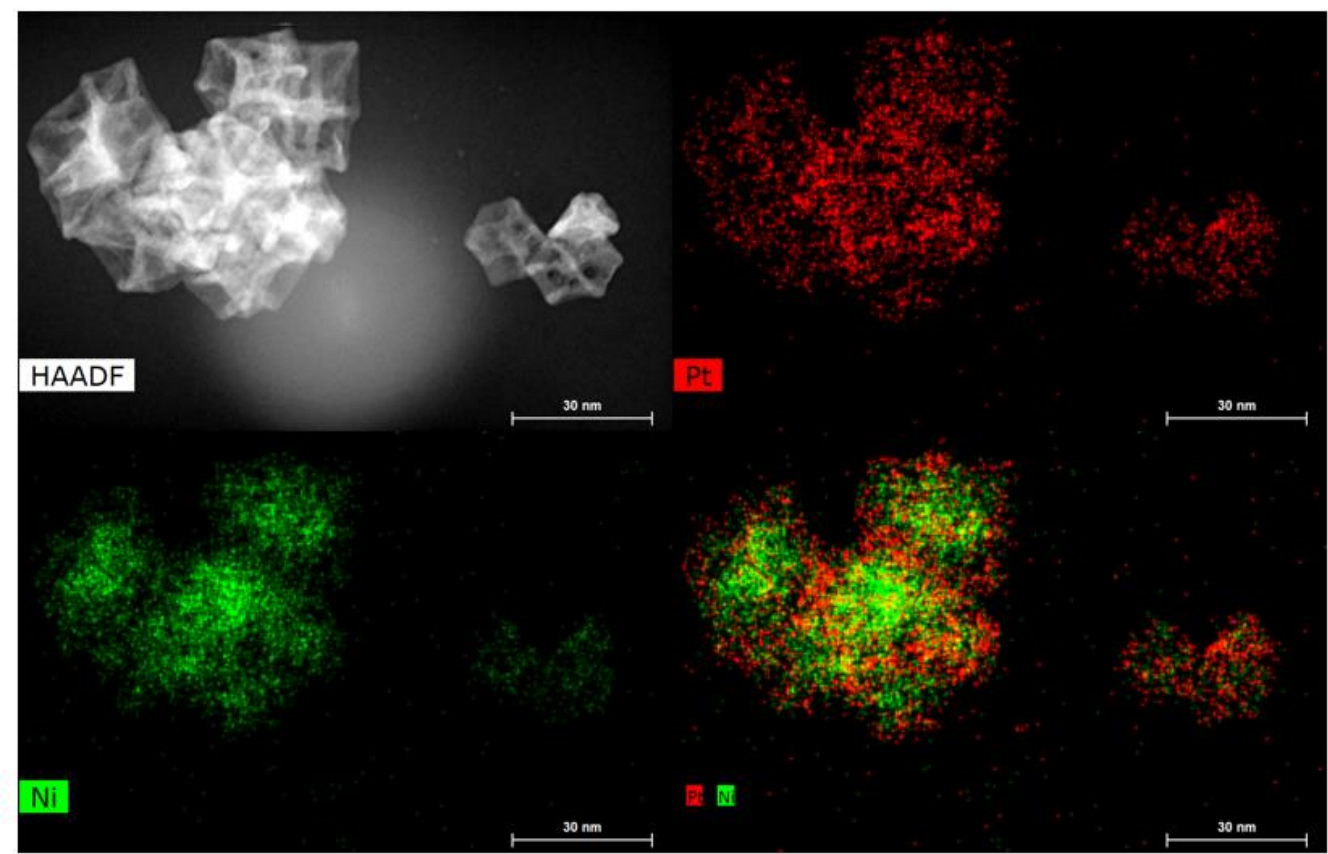

Figure S4. HAADF-STEM images and EDX mapping images of PtNi rhombic dodecahedral nanoframes after $2 \mathrm{hrs}$ of acid etching 


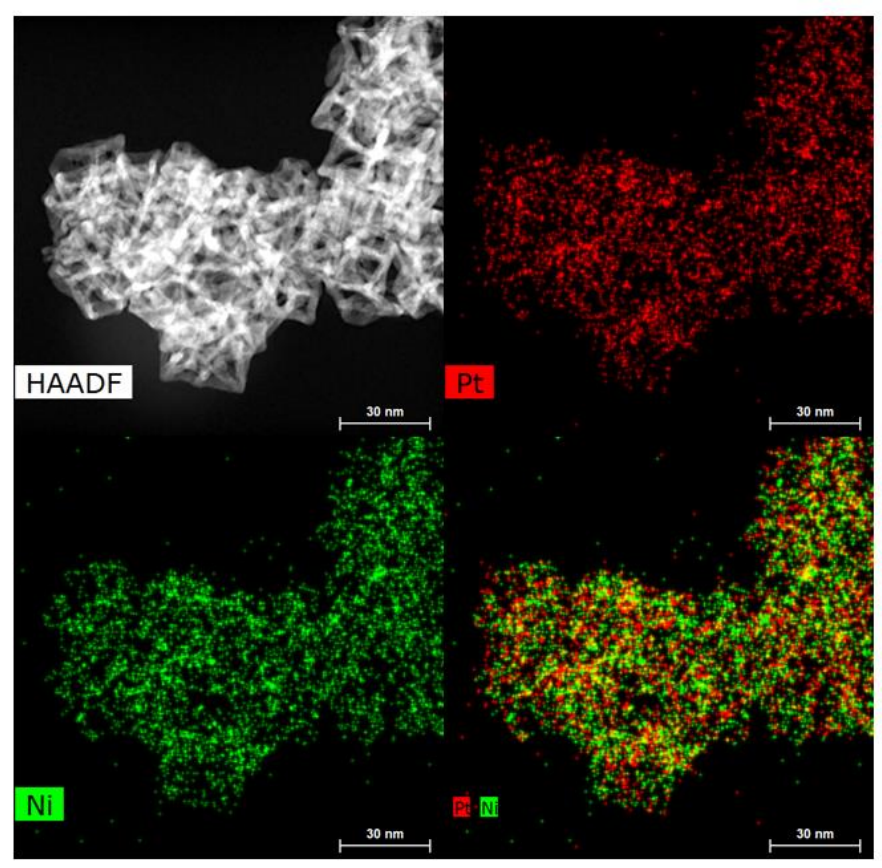

Figure S5. HAADF-STEM images and EDX mapping images of PtNi rhombic dodecahedral nanoframes after 6 hrs of acid etching

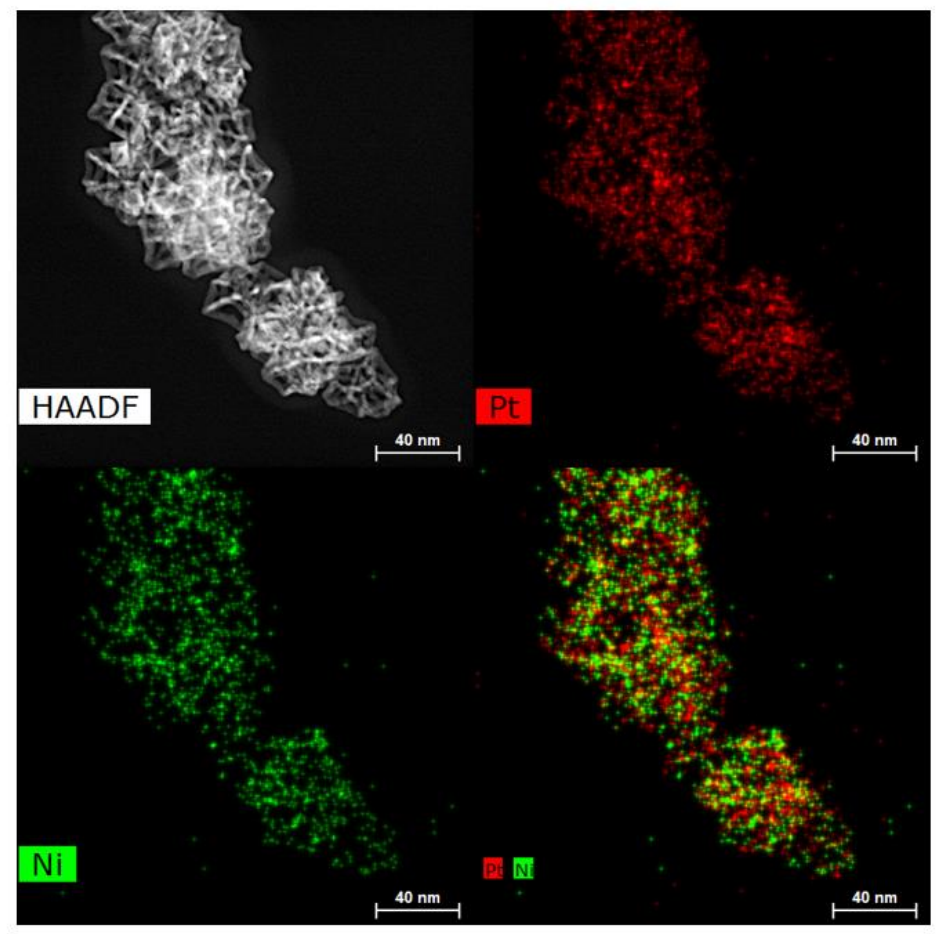

Figure S6. HAADF-STEM images and EDX mapping images of PtNi rhombic dodecahedral nanoframes after $24 \mathrm{hrs}$ of acid etching 


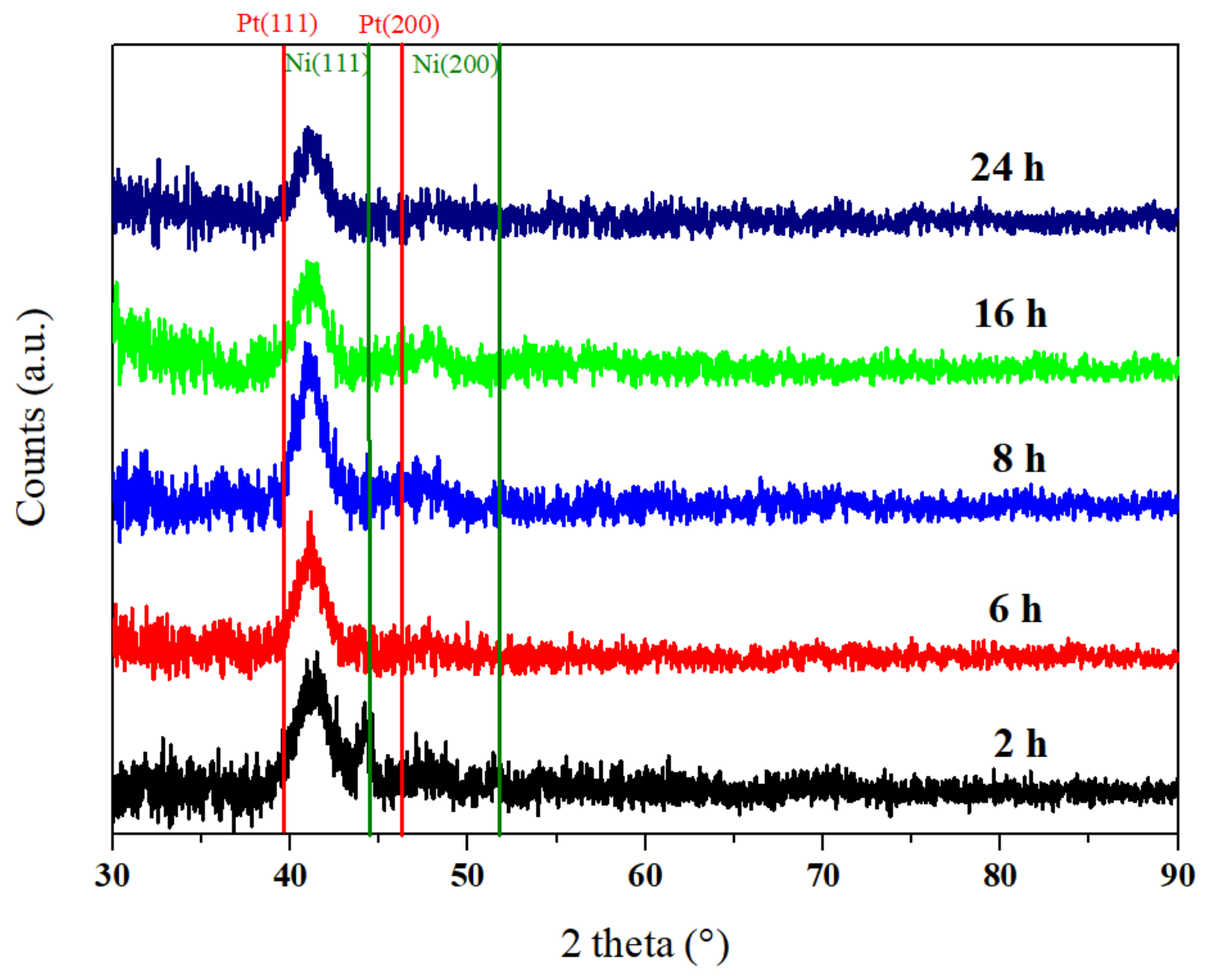

Figure S7. XRD patterns of PtNi nanostructures after a certain duration of oxidative etching process. 


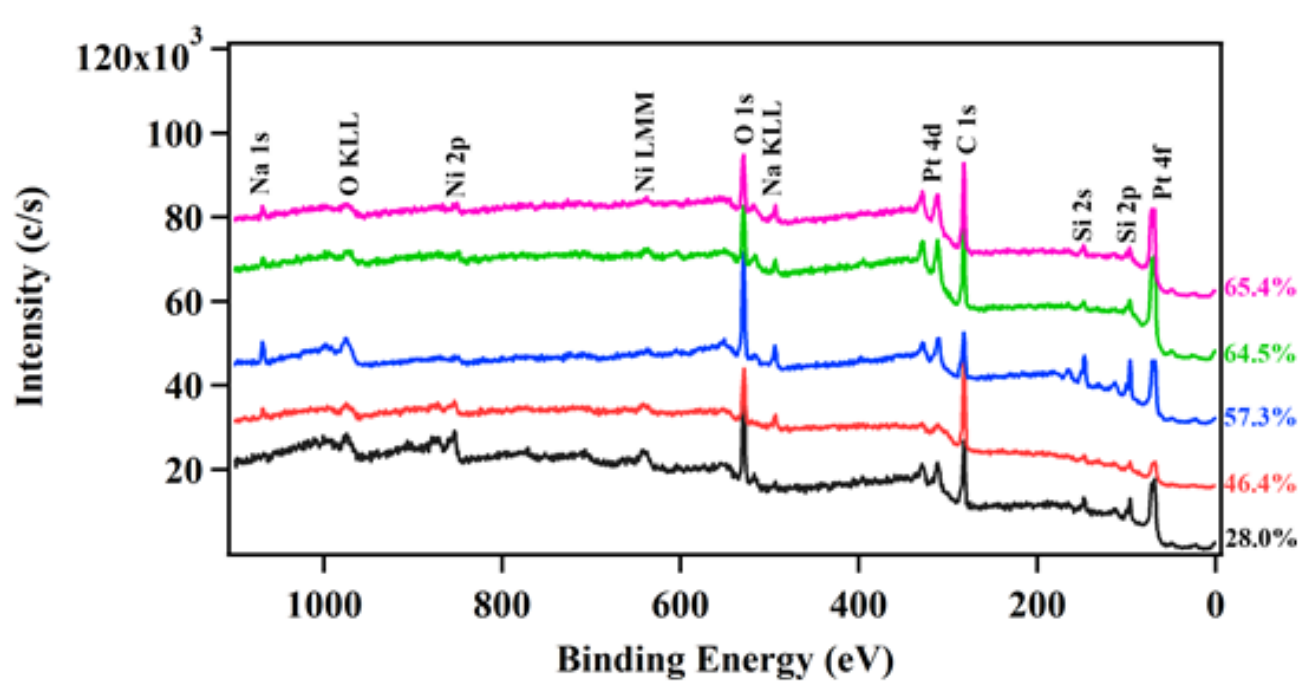

Figure S8. XPS survey spectra of catalyst ranges from $28.0 \% \mathrm{Pt}$ to $65.4 \% \mathrm{Pt}$.
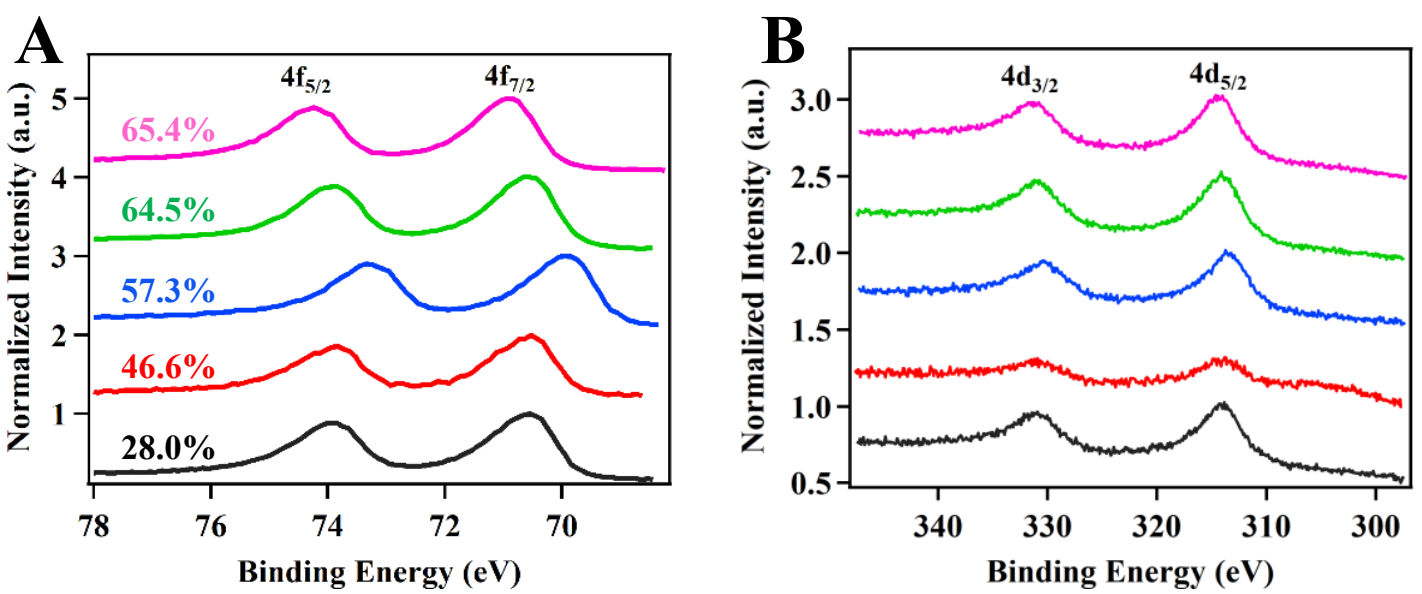

Figure S9. XPS spectra of Pt 4f (A) and 4d (B) of PtNi NF catalysts with compositions ranging from $28.0 \% \mathrm{Pt}$ to $65.4 \% \mathrm{Pt}$.

\begin{tabular}{|c|c|c|c|c|}
\cline { 2 - 5 } \multicolumn{1}{c|}{} & \multicolumn{4}{c|}{ Pt Peak Positions (eV) } \\
\hline \% Pt & $\mathbf{4 f ~}_{\mathbf{7 / 2}}$ & $\mathbf{4 f}_{\mathbf{5} / \mathbf{2}}$ & $\mathbf{4 d}_{\mathbf{5} / \mathbf{2}}$ & $\mathbf{4 d}_{\mathbf{3} \mathbf{2}}$ \\
\hline $28.0 \%$ & 70.5 & 73.9 & 314.2 & 331.4 \\
\hline $46.4 \%$ & 70.5 & 73.9 & 314.0 & 331.5 \\
\hline $57.3 \%$ & 69.8 & 73.1 & 313.6 & 330.7 \\
\hline $64.5 \%$ & 70.3 & 73.6 & 314.2 & 331.3 \\
\hline $65.4 \%$ & 70.6 & 74.0 & 314.5 & 331.7 \\
\hline Average & $70.4 \pm 0.3$ & $73.7 \pm 0.3$ & $314.1 \pm 0.3$ & $331.3 \pm 0.4$ \\
\hline
\end{tabular}

Table S1. Positions of Pt $4 \mathrm{f}$ and $4 \mathrm{~d}$ Peaks 


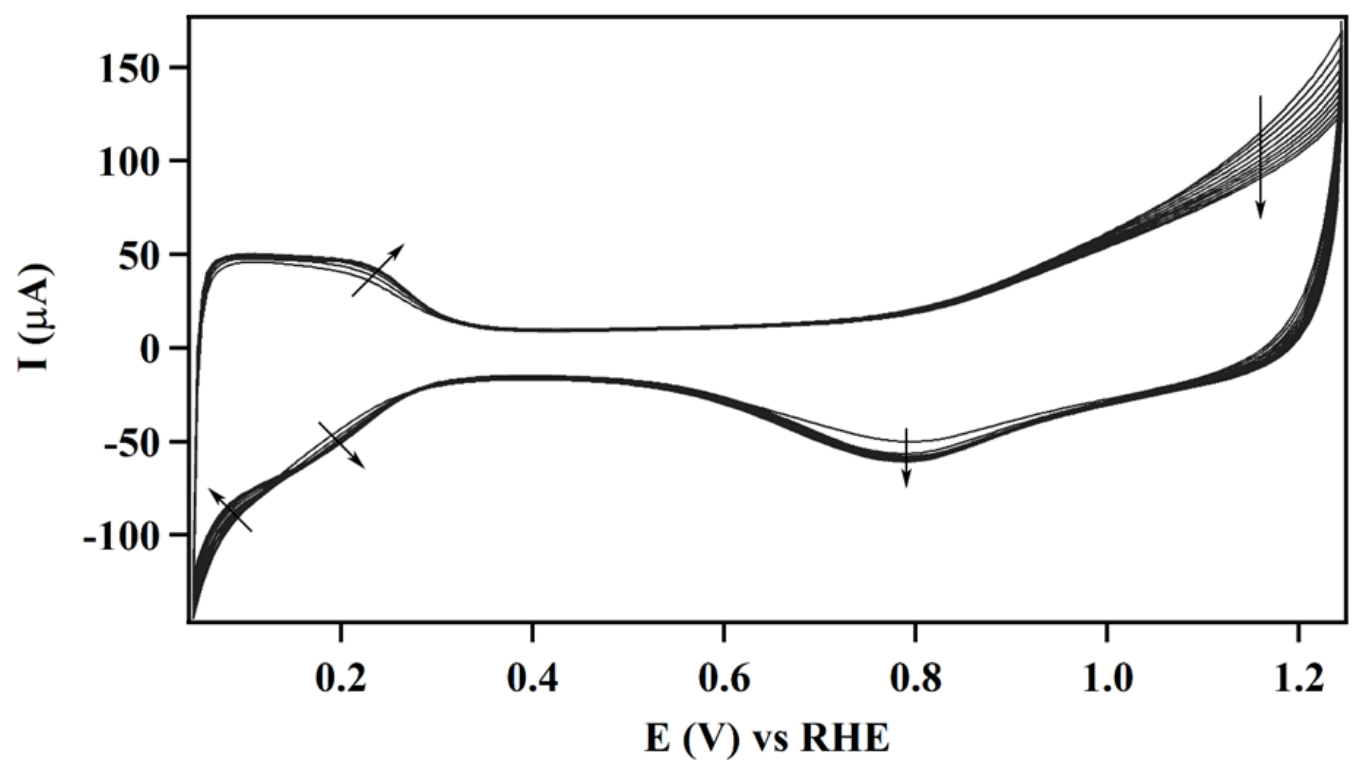

Figure S10. Cyclic voltammogram of $28.0 \% \mathrm{Pt}$ catalyst/C over 25 cycles.

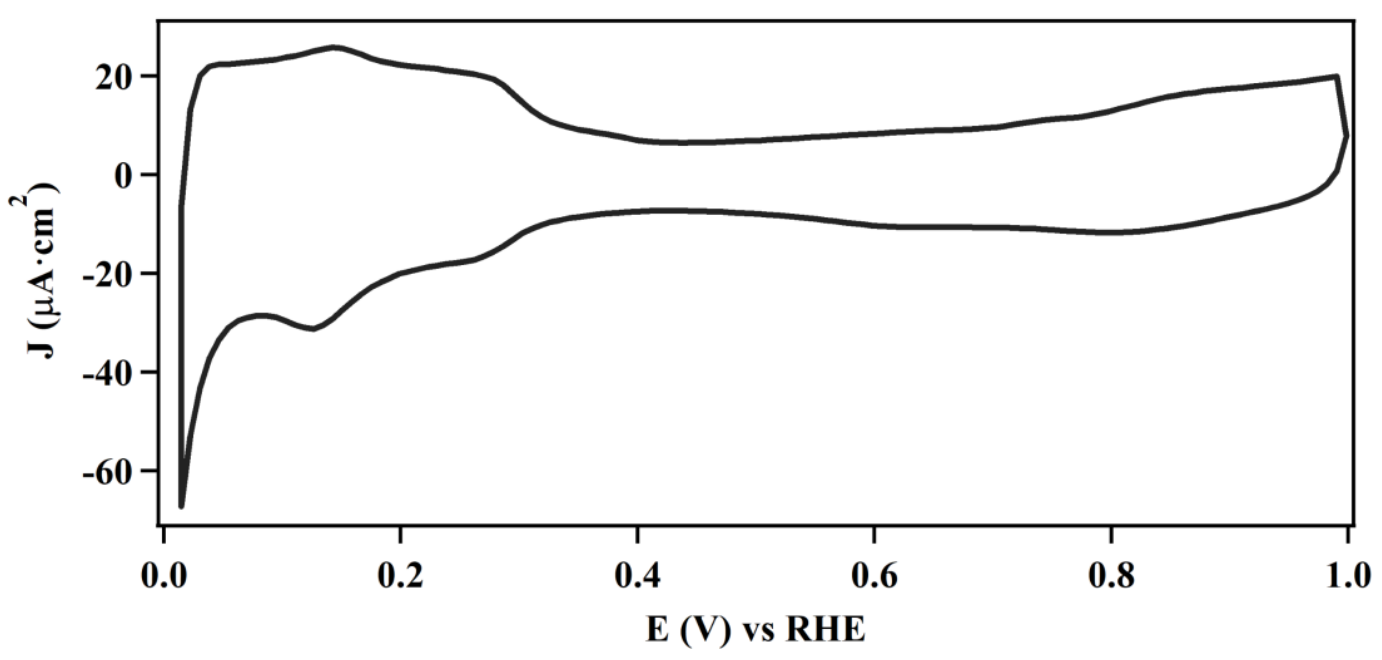

Figure S11. Cyclic voltammogram of Pt NP/C. 


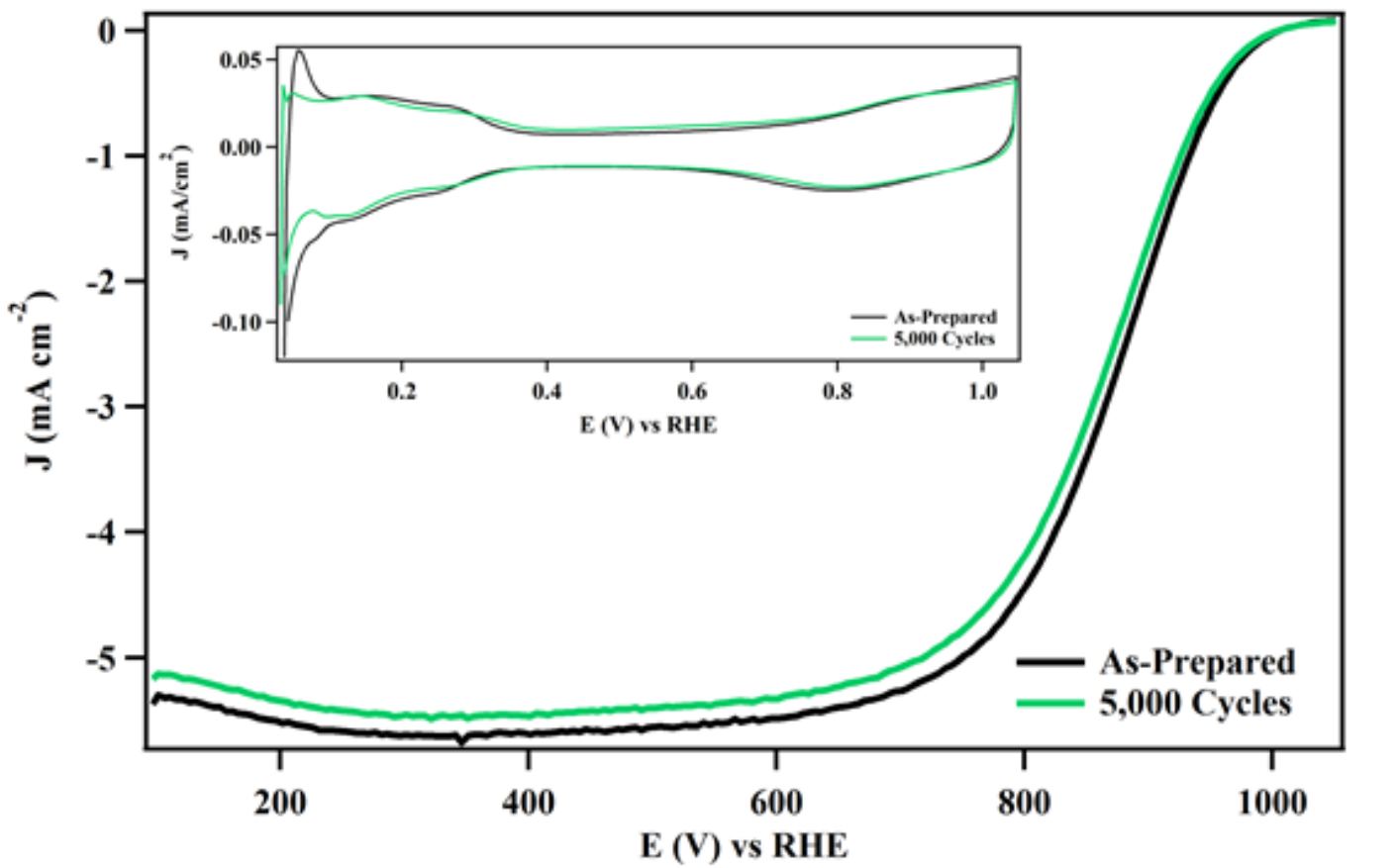

Figure S12. Cyclic voltammogram and ORR polarization curves of the PtNi nanoframe catalyst. 